\title{
COMMENTARY
}

\section{SDGs and the engagement of EU citizens: The role of behavioral science in the energy transition}

\author{
Manuela Amadori ${ }^{1}$ Mariano Votta $^{2^{*}}$ \\ ${ }^{1}$ Active Citizenship Network, EU branch of Cittadinanzattiva APS, Rue Philippe Le Bon 46, 1000 Bruxelles, Belgium \\ ${ }^{2}$ Active Citizenship Network, c/o Cittadinazattiva APS, Via Cereate 6, 00183 Rome, Italy
}

\section{Check for updates}

Correspondence to: Mariano Votta, Active Citizenship Network, c/o Cittadinazattiva APS, Via Cereate 6 , 00183 Rome, Italy;

E-mail: m.votta@cittadinanzattiva.it

Received: March 6, 2021;

Accepted: March 8, 2021;

Published: March 8, 2021

Citation: Amadori M and Votta M. SDGs and the engagement of EU citizens: The role of behavioral science in the energy transition. Resour Environ Econ, 2021, 3(1): 239-244

https://doi.org/10.25082/REE.2021.01.003

Copyright: () 2021 Manuela Amadori, et al. This is an open access article distributed under the terms of the Creative Commons Attribution License, which permits unrestricted use, distribution, and reproduction in any medium, provided the original author and source are credited.

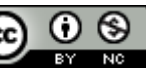

\begin{abstract}
The EU's ambitious targets to increase energy efficiency and cope with the challenge of climate change have pushed several stakeholders, including public authorities and Distributed System Operators (DSOs), to actively invest in the energy transition and improve energy efficiency. Although a significant part of this investment concerns digital infrastructure (i.e., smart meters) allowing citizens to monitor and better manage their consumption of energy, it is pivotal to recognize the necessity for changes in the overall energy-related behavior of consumers. Against this background, the NUDGE project seeks to first analyze the behavior of European citizens with concern the energy consumption and to consequently design and test nudging interventions on different segments of the population, in the hope to derive recommendations tailored to each country and to design more general policies at European level. Hence, pilot projects will be carried out in households, energy communities, and schools in five different EU countries, while a general profiling survey will be disseminated online across the EU. Indeed, in order to profile users and assess the impact of an intervention, NUDGE takes a mixed approach which combines field experiments and randomized control trials with surveys, on-site observations, and reports. The latter provide additional insights to the psychological and contextual variables that result in the behaviors evidenced in the trials. Fundamental to this research is the collaboration of the consortium's partner Cittadinanzattiva-Active Citizenship Network with a number of civic and consumer organizations of different European countries that have shown their interest in the initiative and will collaborate in the dissemination of the online questionnaire in their country. Overall, this project is carried out with the ambition to of raising awareness of the projects' major outcomes among the European institutions, starting from the Inter-Institutional Group "SDG's for well-being and consumers' protection," which was launched at European level in February 2021 with the support of various Members of the European Parliament and the endorsement of 48 European and National Associations.
\end{abstract}

Keywords: energy transition, SDGs, green deal, behavioral science, nudging, civic activism

\section{Introduction}

The EU ambitiously aims to increase energy efficiency and mitigate the negative impacts of climate change by gradually reducing net greenhouse gas emissions and become climate-neutral by 2050 . To achieve this and similar goals, much effort has been devoted to the development of digital infrastructure which allow energy consumer to monitor their energy consumption more accurately and efficiently. Nonetheless, energy efficiency requires radical behavioral changes in the energy-related behavior of consumers, starting from every-day activities such as the way people use heating and electric appliances.

Behavioral science is indeed crucial. Starting from the collection of information that help to understand what determines the choices of individuals allows to inform, design and implement simpler and concrete interventions tailored to the citizens. In addition to informing, behavioral science aims to effectively involve citizens, make them an active part of change, and increase their sense of responsibility with respect to the choices they make in certain situations and contexts. This can be done through targeted communications that stimulate this sense of 
responsibility and of a "healthy competition" in terms of adoption of worthy behaviors compared to other individuals.

Consequently, recent studies have stressed the relevance of nudging interventions, which enable to influence people's behavioral choices in a predictable manner without restricting their options. The notion of "nudging" refers to "any aspect of the choice architecture that alters people's behavior in a predictable way without forbidding any option or significantly changing their economic incentives" [1]. Nudging techniques and more general behavioral interventions can be, and have been, implemented in an array of fields, including the energy sector. However, our understanding of the potential of nudges as means to foster more energy-friendly behaviors remains limited.

Against this background, the project "NUDging consumers towards enerGy Efficiency through behavioral science" (NUDGE) seeks to first analyze the behavior of European citizens with concern the energy consumption and to consequently design and test nudging interventions on different segments of the population [2]. Pilot projects will indeed be carries out in households, energy communities, and schools in five EU countries (Greece, Belgium, Germany, Portugal and Croatia). The research and experimentation of the NUDGE project also aims to develop recommendations tailored to each country and design more general policies at European level.

\section{The project}

Efforts to induce energy-friendly behaviours from end-users through behavioural interventions are characterized by a lack of customer personalization ("one-size-fits-all interventions"), a partial understanding about how different interventions interact with each other and contrasting evidence about their effectiveness, as a result of poor testing under real world conditions. NUDGE has been designed to unleash the potential of behavioural interventions for long-lasting changes in energy friendly behaviours, paving the way to the generalized use of such interventions as a worthy addition to the policy-making toolbox. It takes a mixed approach to the consumer analysis and intervention design with tasks combining surveys and field trials. The project, deeply rooted in behavioral science methods, will investigate individual psychological and contextual variables underlying consumers' attitudes in order to customize the design of behavioral interventions for them, with a strong bias towards nudging-type interventions. The designed interventions are compared against traditional ones in field trials (pilots) in five different EU states, presenting remarkable diversity with regard to innovative energy usage scenarios (e.g., PV production for EV charging, DR for natural gas), demographic and socio-economic variables of the involved populations, mediation platforms for operationalizing the intervention (smart mobile apps, dashboards, web portals, educational material and intergenerational learning practices).

The project NUDGE is characterized by its unique multidisciplinary character, which combines different fields of expertise and capacities. These include knowledge and methods of behavioral science, digital platforms and tools, access to and engagement of heterogeneous user populations, as well as skills in formulating policy recommendations and the capacity to reach and influence policymakers. Therefore, the NUDGE consortium includes partners with highly heterogeneous but remarkably complementary background for the project needs. Overall, the consortium includes ten partners from seven different EU countries and is coordinated by the Institute for European Energy and Climate Policy Stitching (IEECP). The partners are: the Institute For European Energy And Climate Policy (Netherlands); Domx Idiotiki Kefalaiouchiki Etaireia (Greece); Athens University Of Economics And Business Research Center (Greece); Inegi - Instituto De Ciencia E Inovacao Em Engenharia Mecanica E Engenharia Industrial (Portugal); Fraunhofer Gesellschaft Zur Foerderung Der Angewandten Forschung E.V. (Germany); Zelena Energetska Zadruga Za Usluge (Croatia); MVV Energie AG (Germany); Interuniversitair Micro-Electronica Centrum (Belgium); Spring-Stof (Belgium); and Associazione Cittadinanzattiva APS (Italy).

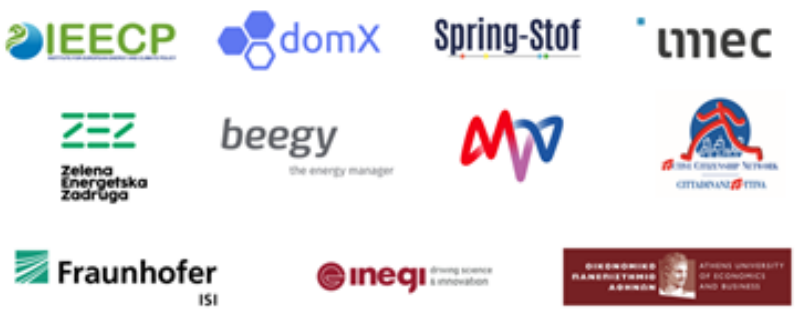


The overarching goal of the NUDGE project is to examine and unleash the potential of behavioral interventions for long-term changes in energy efficiency behaviours, paving the way to the systematic application of such interventions as a worthwhile contribution to policy making. This will be achieved through behavioural interventions; a solid expertise in behavioural science; (mobile) user interface and policy design; synergies between different stakeholders (energy providers, co-operatives, and communities; consumer associations; and technology providers); as well as an extensive network of experts and industrial/consumer associations amplifying the potential for tangible impacts on policy making at all levels. In its effort to achieve its ultimate goal and improve energy efficiency, as well as to overcome the challenges resulting from the implementation of nudging techniques in the energy sector, the project has four major objectives:

(1) Objective 1: Tailor the design of behavioural interventions to individual psychological and contextual variables;

(2) Objective 2: Execute extensive field trials (pilots) that address multiple instances of consumer behaviour, implementing different mixes of behaviour-based and traditional interventions even across participants of the same pilot;

(3) Objective 3: Develop a systematic core and contextual research protocol to continuously measure the impact of the implemented behavioural interventions; and

(4) Objective 4: Consolidate the findings of pilots into recommendations towards policy makers and relevant stakeholders.

The project has received funding from the European Union's Horizon 2020 research and innovation programme under grant agreement No 957012 and has a planned duration of 36 months, starting from September 2020 and ending in September 2023.

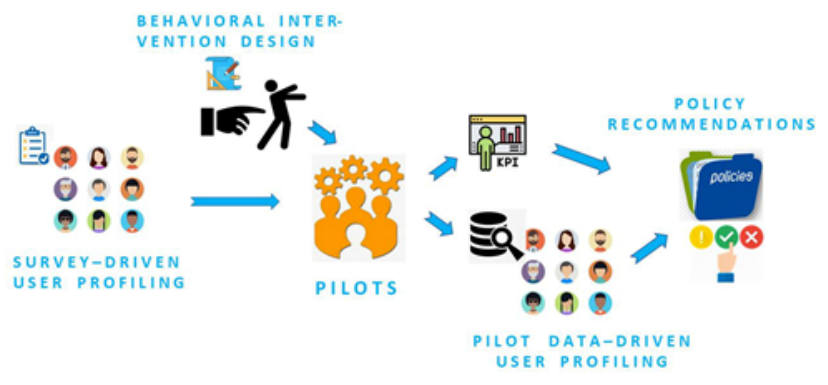

\section{The survey}

One of the first steps of the project is to profile consumers by considering a variety of psychological (e.g., self-efficacy, value priorities, attitudes, outcome expectations) and contextual variables (household size and composition, economic incentives, technologies in use). This will be accomplished through the employment of surveys - realized through online structured questionnaires or interviews - aimed at capturing consumers' perceptions of their own behavior and their original disposition against specific interventions conceived to diminish energy consumption. These insights are pivotal to (a) identify distinct categories/profiles of users; (b) tailor behavioral interventions to these profiles at design level, marking a transition from the "onesize-fits-all" intervention (e.g. nudge) to profile-oriented, or even personalized, interventions; (c) carry out a more-in-depth assessment of interventions' impact on individual user categories, revealing correlations that would otherwise go unnoticed and revealing mechanisms that explain the actual user behaviors, as evidenced in the trials. Hence, survey-type data will be employed in different stages of the project's development.

Indeed, the first phase of the project, which has a planned duration of approximately one year, deals with pre-pilot user profiling based on survey data and aims at collecting and statistically evaluating survey-type data on a diverse set of consumers across Europe. These consumers significantly exceed the number of those participating in the pilot projects and will be engaged through a network of consumer associations and other stakeholders affiliated with "Active Citizenship Network," the EU branch of Cittadinanzattiva. Therefore, during this initial phase, the activities of the consortium will focus on the identification of contextual and behavioral features that are of interest and useful for the user profiling work and consequent preparation of the actual questionnaire (questions, format, web upload); the translation of the questionnaire into a number of different European languages; awareness raising and the dissemination of the survey among a "large enough" population; the promotion of the study among consumers; 
monitoring; the collection and process of data; and the user profiling out of the preprocessed survey data.

The survey will be disseminated among a large set of consumers, including both pilot participants and external consumers involved through consumer associations contributing to the project, to collect basic contextual (i.e., age, income, household composition, house area) and socio-cognitive (perception of energy use, self-efficacy, goals) information about them. These surveys will be used to record and evaluate the running behaviors of the pilot participants about energy-efficiency measures and actions; analyze the reactions of end users to the various behavioral interventions and derive predictive models of their energy-related behavior; classify different groups of end users on the basis of their energy-related behavior and explore their generalizability across pilots.

The objective is to gather information about the following aspects at household level:

(1) Household characteristics (income, number and age of members), building characteristics (type, area, energy-friendliness), and level of environmental conscience.

(2) Consumer behavior with respect to the energy consumption aspects. This pertains to the ownership and use of heating devices, the overall home power consumption, the use of EV charging stations.

(3) Their intentions and perceptions with regard to the adoption of measures that enhance the energy efficiency. For instance, the installation of PV, the optimization of heating device settings in automated or manual way or their alleged response to incentives such as discounts and bonuses for changing their behavior towards more energy-efficient profiles.

(4) The existence of extrinsic factors such as market factors or laws but also digital illiteracy or privacy/security-related concerns related to the use of smart devices that stand (or are perceived as) major obstacles to the adoption of more energy efficient behaviors.

Remarkably, this questionnaire does not have a specific target of consumers, as the NUDGE project addresses all European consumers, of any gender, age, social status, etc. For this reason, the questionnaire is written in a clear and simple way so that anyone can answer the questions reflecting on their daily habits and their awareness about energy consumption and saving. Unlike the questionnaire, the five pilot projects that will be developed during 2021-2022 (in five European countries) have different and well-identified targets, allowing the consortium to analyze the behavior of different groups of European consumers and demonstrate effective interventions in different energy consumption scenarios.

To ensure the effective outcome of this first phase, spreading awareness of the survey is not enough. Instead, a good, coordinated effort to disseminate the survey as widely as possible and ensure participation of end users is pivotal. This will be possible thanks to the contribution of the civil society, especially European consumer associations. Indeed, fundamental to this research is the collaboration of the consortium's partners Cittadinanzattiva-Active Citizenship Network with a number of civic and consumer organizations of different European countries that have shown their interest in the initiative and will collaborate in the translation and dissemination of the online questionnaire in their country.

The questionnaire is available online in the following languages: English, Dutch, French, Italian, Portuguese, Croatian, Greek, German, Lithuanian, Latvian, Romanian, Slovenian, Slovak, and Spanish. It will be distributed online by the members of the NUDGE consortium, including the Italian NGO Cittadinanzattiva, as well as other consumer organizations in several European countries. The surveys in the different languages can be accessed on Active Citizenship Network's website [3].

\section{The role of the civil society towards a more sustain- able Europe}

A large part of EU citizens is now aware of climate change issues and of the energy transition, and they are willing to make correct choices in their energy consumption. However, the gap between intentions and concrete actions remains wide. Prior to the Covid-19 pandemic, young people in several EU countries called for a more ambitious political leadership on climate action, sustainable choices, and green lifestyles. In this regard, the New Consumer Agenda - adopted in November 2020 by the European Commission - gives momentum to the EU consumer policy, which aims to better equip the EU to face new challenges for consumers' rights and to welcome new opportunities for consumers' empowerment in a time characterized by the green and digital transitions, the COVID pandemic and the post-COVID recovery [4]. Therefore, it is time for all citizens to be united by the language of the SDGs and to actively participate in the transition to a green economy, thereby transforming their "good intentions" in a shared "culture of doing." In 
this context, the NUDGE project recognizes the key role of citizens in the energy sector, as their behavior is crucial to promote energy efficiency and thus a low-carbon, climate resilient future. In order to attain Objective 7 of the UN's 2030 Agenda for Sustainable Development, which aims at "ensuring access to economic, reliable, sustainable and modern energy systems for al", not only energy sources need to become more 'renewable', but also consumers' behaviours and choices [5].

Since the energy transition starts from the citizens, they have to be directly involved in initiatives targeting their attitudes and everyday life. To encourage citizens' participation, as well as to guarantee a significant participation of civic actors in the NUDGE project, Cittadinanzattiva has involved different external stakeholders, including civic and consumer organizations, but not only. These will assist Cittadinanzattiva and the other members of the consortium in the first phase of the project by helping translate the general profiling survey in their native language and disseminate it across their country. These external stakeholders include: the Croatian Association for Consumer Protection (Croatia) [6], the Center for education and informing consumers (Croatia) [7], the association InfoCons (Romania) [8], the Union of Working Consumers of Greece (Greece) [9], the Association of Consumers Organizations in Slovakia (Slovakia) [10], the association Talented Borders (Latvia) [11], the Confederation of Consumers and Users (Spain) [12], IFOK (Germany) [13], the National Association Saugok Save (Lithuania) [14], the Consumer Rights Protection Centre (Lithuania) [15], Indecosa (France) [16], the Consumers Association of Malta (Malta) [17], Association for Consumer Rights (Malta) [18], Consumur (Spain) [19], Social-Mentes Canarias (Spain) [20], the Slovene Consumer Association - ZPS (Slovenia) [21], and the European Consumer Union through its member organizations [22].

Moreover, Cittadinanzattiva has established different media partnership at international, regional, and national level. The media partners include The Innovation Platform [23], the peer-reviewed journal Resources and Environmental Economics [24], EKOenergy [25], and the Italian online magazine Canale Energia [26].

Thanks to the contribution of these stakeholders, as well as of the members of the NUDGE consortium, the questionnaire is available in 14 European languages. It will be distributed through the use of online magazines and journals, social media platforms, websites, newsletters, and emails by the members of the NUDGE consortium, including the Italian NGO Cittadinanzattiva, as well as other consumer organizations in several European countries.

\section{Conclusion}

The transition to a sustainable and efficient economy requires radical changes in the consumption habits of our society Inter-Institutional Group SDGs for well-being and consumers' protection and in the production models of our economy. As stated by the European Commission's President Ursula Von Der Leyen in her presentation of Europe's roadmap for a green transition [27], the transition to a sustainable Europe has to be based on the commitment that "no one is left behind," and that everyone has to be involved in the achievement of this goal. "Leaving no one behind" also means empowering as many people as possible to play a positive and active role so that they can fully participate in the transition. For these reasons, the active participation of European citizens is pivotal for this transition. Consequently, the consortium hopes that the results of the NUDGE project will be taken into consideration during EU debates hosted in the framework of those citizen agoras suggested by the European Parliament [28]. Accordingly, the main outputs to the project will be presented to the Inter-Institutional Group "SDGs for well-being and consumers' protection" [29], promoted by Cittadinanzattiva through its EU branch Active Citizenship Network (ACN), the European Consumers Union (ECU) and the Italian Alliance for Sustainable Development (ASviS). This group was indeed created to become a) an awareness raising catalyst across Europe for civil society, business, research, media, etc at national and European level; b) a dialogue facilitator between the European institutions and stakeholders active in specific fields; c) an incubator of good practices in order to facilitate the process of exchange, contamination and dissemination of best practices experiences to enable EU citizens to make sustainable choices.

\section{References}

[1] Thaler R and Sunstein C. Nudge: Improving Decisions About Health, Wealth, and Happiness. Rev. and expanded ed. New York: Penguin Books, 2009. 
[2] NUDGE. N.d.

https://www.nudgeproject.eu

[3] NUDging consumers towards enerGy Efficiency through behavioral science (NUDGE). Join the Survey. 2021. Active Citizenship Network.

http://www.activecitizenship.net/consumers-rights/news/381-nudging-consumers-towards-energy -efficiency-through-behavioral-science.html

[4] New Consumer Agenda: European Commission to empower consumers to become the driver of transition. 13 Nov. 2020. European Commission. https://ec.europa.eu/commission/presscorner/detail/en/ip_20_2069

[5] The Sustainable Development Agenda. N.d. United Nations. https://www.un.org/sustainabledevelopment/development-agenda

[6] Croatian Association for Consumer Protection (Hrvatska Udruga Za Zastitu Potrosaca - HUZP). https://www.consumersinternational.org/members/members/croatian-association-for-consumer-pro tection-cacp

[7] Center for education and informing consumers (Centar za edukaciju i informiranje potroaa - CEIP). https://www.ceip.hr

[8] InfoCons (Asociaia InfoCons - Organizaie pentru Protecia Consumatorilor). https://www.infocons.ro

[9] Union of Working Consumers of Greece. https://eeke.gr/en

[10] Association of Consumers Organizations in Slovakia (Asocicia spotrebiteskch subjektov Slovenska). http://www.spotrebitelinfo.sk

[11] Association Talented Borders. https://ashandurov.wixsite.com/talentedborders

[12] Confederation of Consumers and Users (Confederacin de consumidores y usuarios CECU). https://cecu.es

[13] IFOK.

https://www.ifok.de

[14] National Association Saugok Save. https://www.facebook.com/saugoksave

[15] Consumer Rights Protection Centre (Asociacija Vartotoju teisiu gynimo centras). http://www.vartotojucentras.lt

[16] Indecosa. http://www.indecosa.cgt.fr

[17] Consumers Association of Malta (Ghaqda tal-Konsumaturi). http://camalta.org.mt

[18] Association for Consumer Rights (ACR). http://www.acrmalta.com

[19] Asociacin Murciana de Consumidores y Usuarios (CONSUMUR). http://consumur.org

[20] Social-Mentes Canarias. https://www.facebook.com/Social-Mentes-Canarias-175786197355387

[21] Slovene Consumer Association (Zveza Potronikov Slovenije ZPS). https://www.zps.si

[22] European Consumer Union. https://europeanconsumersunion.eu/?lang=en

[23] The Innovation Platform. https://www.innovationnewsnetwork.com/the-innovation-platform

[24] Resources and Environmental Economics. https://www.syncsci.com/journal/REE

[25] EKOenergy. https://www.ekoenergy.org

[26] Canale Energia. https://www.canaleenergia.com

[27] Turning challenge into opportunity on the course to becoming the first climate-neutral continent. 13 Dec. 2019. European Commission. https://ec.europa.eu/commission/presscorner/detail/en/AC_19_6778

[28] European Parliament resolution of 15 January 2020 on the European Parliaments position on the Conference on the Future of Europe (2019/2990(RSP). https://www.europarl.europa.eu/doceo/document/TA-9-2020-0010_EN.html

[29] Inter-Institutional Group Sustainable Development Goals for well-being and consumers protection Official Website. n.d.

http://www.interestgroupsdgs.eu 